Review

\title{
Research Progress of Moyamoya Disease in Children
}

\author{
Jianmin Piao, Wei Wu, Zhongxi Yang, Jinlu Yu ${ }^{凶}$ \\ Department of Neurosurgery, First Hospital of Jilin University, Changchun, 130021, P.R. China \\ $\triangle$ Corresponding author: Jinlu Yu, Department of Neurosurgery, First Hospital of Jilin University, 71 Xinmin Street, Changchun, 130021, \\ P.R. China. Email: jinluyu@hotmail.com \\ (C) 2015 Ivyspring International Publisher. Reproduction is permitted for personal, noncommercial use, provided that the article is in whole, unmodified, and properly cited. \\ See http://ivyspring.com/terms for terms and conditions.
}

Received: 2015.01.28; Accepted: 2015.06.02; Published: 2015.07.03

\begin{abstract}
During the onset of Moyamoya disease (MMD), progressive occlusion occurs at the end of the intracranial internal carotid artery, and compensatory net-like abnormal vessels develop in the skull base, generating the corresponding clinical symptoms. MMD can affect both children and adults, but MMD in pediatric patients exhibits distinct clinical features, and the treatment prognoses are different from adult patients. Children are the group at highest risk for MMD. In children, the disease mainly manifests as ischemia, while bleeding is the primary symptom in adults. The pathogenesis of MMD in children is still unknown, and some factors are distinct from those in adults. MMD in children could result in progressive, irreversible nerve functional impairment, and an earlier the onset corresponds to a worse prognosis. Therefore, active treatment at an early stage is highly recommended. The treatment methods for MMD in children mainly include indirect and direct surgeries. Indirect surgeries mainly include multiple burr-hole surgery (MBHS), encephalomyosynangiosis (EMS), and encephaloduroarteriosynangiosis (EDAS); direct surgeries mainly include intra- and extracranial vascular reconstructions that primarily consist of superficial temporal artery-middle cerebral artery (STA-MCA) anastomosis. Indirect surgery, as a treatment for MMD in children, has shown a certain level of efficacy. However, a standard treatment approach should combine both indirect and direct procedures. Compared to MMD in adults, the treatment and prognosis of MMD in children has higher clinical significance. If the treatment is adequate, a satisfactory outcome is often achieved.
\end{abstract}

Key words: Children; Moyamoya disease; surgical treatment; prognosis

\section{Introduction}

MMD was first described in the Japanese litera-

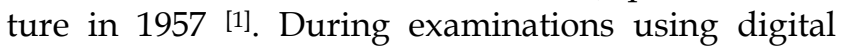
subtraction angiography (DSA), computed tomographic angiography (CTA), and magnetic resonance angiography (MRA), a stenosis or occlusion was discovered at the end of the internal carotid artery, and compensatory abnormal proliferation of the vascular network was observed in the skull base [2]. MMD shows two peaks of onset in the human population: one at approximately 40 years of age, which manifests as bleeding during clinical onset, and another at approximately five years of age, which manifests mainly as clinical ischemia, also known as MMD in children [3]. The severity of MMD in children is age-related; in younger children, the condition is more severe. Stud- ies have shown that children younger than four years often exhibit poor prognoses in MMD [4]. The incidence of MMD shows obvious regional differences. MMD is more commonly observed in Eastern Asian countries, such as Japan, Korea, and China. In Japan, the incidence of MMD is 3-10/100,000, whereas the incidence is $0.09 / 100,000$ in the USA [5]. MMD is more common in female patients, and the male to female ratio ranges from $1: 1.8$ to $1: 4.25[6,7]$. Currently, no specialized literature has reported the epidemiology of MMD in children, but pediatric patients have been included in epidemiological analyses of MMD, which suggests that MMD in children might also be consistent with the epidemiological characteristics described above. 
The pathogenic mechanism of MMD in children has not been clarified. Based on a review of the relevant literature, the pathogenesis of MMD could be related to genetic, immune, and radiological factors, along with the abnormal expression of angiogenesis factors. In addition to MMD, we also reviewed studies of children with Moyamoya syndrome (MMS) due to the many similarities between MMD and MMS. MMS is often pertinent to many other conditions, such as multiple neurofibromatosis, head radiotherapy, sickle cell anemia, and Down's syndrome [8,9]. DSA is the gold standard for MMD diagnosis. However, due to the young age of affected children, the application of DSA is limited. Therefore, many other auxiliary examination methods, such as MRA and CTA, have also been applied. MRA and CTA have high specificities in MMD diagnoses [10]. The treatment of children with MMD also has its own characteristics, primarily because the vessels on the brain surface are slender and fragile, and therefore, direct vascular reconstruction is much more difficult in children than in adults. In addition, the blood supply area of the branches of the superficial temporal artery (STA) is limited so that functional cortices are often not covered. Therefore, the application of indirect surgery has been extensive [11]. Indirect surgery for children with MMD includes surgeries such as multiple burr-hole surgery (MBHS), encephalomyosynangiosis (EMS) and encephaloduroarteriosynangiosis (EDAS). Previous studies have reported that these procedures demonstrate better effects in children than in adults [12]. In fact, no standard treatment plan for children with MMD is currently applied in clinical practice [13].

\section{Possible pathogenic mechanisms of MMD in children}

The symptoms of children with MMD are primarily the result of progressive vascular stenosis. The histological characteristics of vascular stenosis include fibrointimal hyperplasia, impairment of the elastic layer of the media, and vascular smooth muscle cell proliferation [14]. A previous study reported that the small irregular wave-shaped changes at the internal elastic membrane could be regarded as histological characteristics of MMD [15]. However, which mechanism results in the discussed pathological changes in children with MMD has yet not been determined. Based on a literature review, the following factors were determined to be influential in the MMD pathogenesis of children.

\section{Genetic factors}

The genetic influence of MMD is primarily due to its familial distribution. Previous studies have revealed that $10 \%$ of MMD cases occur in the same family, and $70 \%$ occur in siblings, which involves multiple pediatric patients [16]. The genetics of MMD appear to involve a multiple genetic factor principle. Papavasiliou et al. studied the genotypes of two European children with MMD and discovered that the MT3 sequence in the D-loop of the mitochondrial DNA and the $Y$ chromosome gene in these European patients are consistent with the genetic sequences found in Japanese MMD patients. These results indicated that these genes might be correlated with MMD onset [17]. HS Kang et al. discovered that the G/C hybrid genotype at position 418 of the TIMP2 promoter is an inducer of familial MMD [18]. Sakurai et al. revealed that autosome 8q22.3, which encodes the TIEG genotype, could be the genetic sequence that causes MMD [19]. Fujimura et al. found that RNF213 was an important predisposing gene for MMD in eastern Asian children [20]. These studies did not exclude childhood MMD cases. Therefore, MMD in children may also include genetic variations, as discussed above.

$\mathrm{SH}$ Hong et al. showed that, in the children with MMD, the allelic genes of HLA-DRB1*1302 and DQB1*0609 exhibit correlations with MMD occurrence. This study further suggested that a genetic polymorphism in the HLA-Class-II genome was the inducing factor of familial MMD [21]. YS Park et al. discovered that the allelic gene of VEGF-634G was related to MMD in children [22]. Hitomi et al. found that the RNF213 R4810K mutation induced mitotic abnormalities and increased the risk of genomic instability. Ultimately, its polymorphism could increase susceptibility to MMD, including pediatric MMD [23]. Furthermore, the RNF213 R4810K mutation reduced the angiogenic activity of induced pluripotent stem cells (iPSECs) in patients with MMD, suggesting that it may be a promising in vitro model for MMD [24]. In a rare case of a mutation of CBL associated with MMD, Hyakuna et al. showed that both NF1 and CBL participate in the RAS/MAPK pathway, indicating a common underlying pathogenesis of MMD [25]. Darrigo Júnior LG et al. examined a pediatric patient with multiple neurofibromatosis and discovered that a mutation of the NF-1 gene could stimulate the abnormal protein function of neurofibromatosis, causing vascular smooth muscle cell proliferation and resulting in MMS [26]. It is worth noting that due to differences in clinical symptoms observed between children and adults with MMD, the genotypes of the two populations might also be different [27].

\section{Abnormal angiogenesis factors}

A previous study revealed that vascular endothelial growth factor (VEGF) could be one of the causes of MMD by testing cerebrospinal fluid and 
blood from MMD patients [28]. In addition, Yoshimoto et al. found increased concentrations of basic fibroblast growth factor (bFGF) in cerebrospinal fluid in children with MMD. bFGF could induce vascular endothelial cell, smooth muscle cell, and fibroblast proliferation and angiogenesis, which suggested that bFGF could be a predisposing factor for MMD in children ${ }^{[29]}$. Hojo et al. showed that the serum concentration of transforming growth factor-beta1 (TGFb1) was extremely high in children with MMD. TGFb1 plays an important role in cell proliferation and mutation and regulates the expression of connective tissue genes and angiogenesis, suggesting that childhood-onset MMD may be related to TGFb1 [30].

\section{Infection immune factors}

The human immune response to the invasion of microorganisms has also been suggested to cause MMD [31]. Yamada et al. found high levels of Propionibacterium acnes (P. acnes) antibody titers in the sera of MMD patients, suggesting that $P$. acnes and immune factors may be related to the occurrence of MMD in both children and adults [32]. Sharfstein et al. discovered that the human immunodeficiency virus (HIV) could introduce vascular lesions in the brain, which could result in MMD. However, this study only included adult patients. Further study is required to confirm whether HIV infection could result in MMD in children [33]. However, Hsiung GY et al. reported a 10-year-old MMD patient in whom the cause of onset was related to congenital HIV infection [34]. TK Kim et al. reported an acute necrotizing encephalopathy (ANE) case that occurred on the $24^{\text {th }}$ day after birth, which was cured. Twenty-eight months after birth (27 months after the encephalopathy was cured), the patient suffered from sudden awareness and movement disorders and was diagnosed with childhood-onset MMD after a DSA examination [35]. $\mathrm{H} \mathrm{Li}$ et al. examined 114 childhood-onset MMD cases and found that enhanced thyroid function and increased thyroid autoantibody levels were closely related to MMD development [36]. These results suggested that the occurrence of childhood-onset MMD might be pertinent to infections and immune responses.

\section{Radiation-inducing factors}

Radiation treatment has also been suggested as a factor in the development of childhood-onset MMD. Radiation therapy can degrade both vascular walls and the elastic tissue on the inner walls of blood vessels and can cause occlusions at the end of the internal carotid artery, resulting in MMD [37]. Kikuchi et al. discovered that frequent head radiation treatments in acute pediatric patients with lymphoblastic lymphoma could induce MMD [38]. Ullrich et al. revealed that radiation treatment for intracranial tumors in children could also result in MMD. In addition, it has been observed that in younger patients that receive higher dosages of radiation, the probability of developing MMD is increased [39]. Kestle et al. examined 28 children with optic glioma and found that after receiving radiation treatment, five patients developed MMD after surgery. In contrast, none of the 19 patients who did not receive radiation therapy developed MMD [40]. This difference may have been observed because radioactive treatments can result in MMD-like variations in the blood vessels in the brain of patients with affirmative causes. These cases can also be regarded as MMS.

\section{Other factors}

In addition to the pathogenesis mechanisms discussed above, other studies have also mentioned several pathogenic factors of MMD that are primarily related to MMS. Therefore, it is necessary to define Moyamoya syndrome. It is an MMD-like disease induced by other diseases (such as multiple neurofibromatosis, sickle cell anemia, Down's syndrome, Grave's disease, and hemoglobin Southampton) or adverse factors (such as radiation therapy or chemotherapy) [41,42,43]. For example, Buchbinder et al. performed interferon-alpha chemotherapy while treating a 12-year-old osteosarcoma patient and discovered that the patient's intracranial vasculature exhibited serious MMD-like changes. This result suggested that interferon-alpha might be correlated with MMD development ${ }^{[44]}$. Meena SS et al. reported the MRA results of a 3-year-old patient with severe iron deficiency anemia with MMD-like lesions, thus implying a correlation between the pathogeneses of the two diseases [45]. Vo Van $\mathrm{P}$ et al. reported a 5-year-old patient with hereditary spherocytosis. The patient received an MRA exanimation due to a transient ischemic attack (TIA) and was found to have intracranial MMD-like lesions [46]. Ramesh $\mathrm{K}$ et al. examined a 14-month-old infant with MMD and suggested that the onset was related to renal agenesis and external iliac artery stenosis. The authors came to the conclusion that the patient had developed MMD while in the uterus [47].

\section{Diagnosis of MMD in children}

\section{Diagnostic criteria}

In 1997, the Research Committee on MMD published the first English guidelines for MMD diagnosis and treatment. An MMD diagnosis should satisfy the following three criteria: stenosis or occlusion appears at the end of the internal carotid artery; an abnormal vascular network (known as MMD vasoganglion) is present; and bilateral changes occur [48]. The diagnos- 
tic criteria for MMD in children are slightly different from those of adults. In children, an MMD diagnosis can be confirmed if a unilateral change is observed. In other words, both unilateral and bilateral changes can be diagnosed as childhood-onset MMD; however, in adult cases of MMD, the changes must be bilateral. If a unilateral MMD-like change is observed in an adult, it can only be diagnosed as possible or unilateral MMD. However, the diagnostic criteria of MMS are the same for both children and adults. Specifically, relevant diseases are confirmed based on the diagnostic criteria discussed above (i.e., risk factors that result in the vascular changes discussed above) [49].

\section{Diagnostic methods.}

The imaging diagnosis and evaluation of children with MMD include DSA, MRA (MRI), CTA (CT), cerebral perfusion imaging, and electroencephalography (EEG), among others.

\subsection{DSA}

DSA is the gold standard for the development of a diagnosis and treatment plan before surgery in children with MMD. In addition, DSA has decisive implications for follow-up and post-surgical vascular reconstruction evaluations. During the diagnosis of childhood-onset MMD, in addition to the basic characteristics of stenosis and the occultation of the internal carotid artery and its major branches, the extensive formation of a collateral circulation bypass is also an important diagnostic factor. DSA can provide information regarding the hemodynamic mode. A collateral circulation bypass can be classified into the following four types: (1) basal surface of the cerebral vascular network; (2) extensive expansion of the anterior choroidal artery and the posterior pericallosal artery; (3) the ethmoidal artery vascular network; and (4) the parietal vascular network [50]. When evaluating the degree of post-surgical vascular reconstruction, Matsushima et al. defined classification standards according to DSA to evaluate the formation of collateral circulation induced by vascular anastomosis. Grade A indicates that over two-thirds of the middle cerebral artery (MCA) anastomosis-induced circulation is fulfilled, grade B indicates one-third to two-thirds fulfillment, and grade $C$ represents less than one-third fulfillment [51]. Considering the special treatment parameters for children, the application of DSA also exhibits obvious drawbacks, such as invasiveness and radiation-induced harm to the body.

\subsection{MRA and CTA}

Even though the accuracies of MRA and CTA are not as high as that of DSA, these techniques are still extensively used in the diagnosis of MMD in children. MRA in particular has become a screening tool for the identification of high-risk MMD-affected children [52]. The primary reason for this preference is that MRA causes no radiation damage in pediatric patients and achieves an ideal accuracy in diagnosis. For example, Yamada et al. examined the accuracy of MRA in the diagnosis of intracranial artery stenosis and discovered that, compared to DSA, MRA could achieve $88 \%$ accuracy in the internal carotid artery (ICA), 83\% accuracy in the anterior cerebral artery (ACA), and 88\% accuracy in the MCA, which suggested that the sensitivity and specificity of MRA for artery stenosis were close to $100 \%$ [53]. MRA is not only suitable for the diagnosis of MMD in children, but is also applicable for both treatment and follow-up after treatment. For example, continuous MRA examinations can trace the post-surgical vascular reconstruction process. Houkin et al. used MRA to observe that an MMD vascular lesion began to degrade one month after vascular bypass surgery. Meanwhile, the diameters of the deep temporal artery and middle meningeal artery increased accordingly. All of these transitions were observed on MRA within three months after the surgery [54]. The application of CTA in children with MMD is far less common than in adults, which might be due to the requirement of a contrast medium and the physical control required during the time window of image acquisition. However, compared to MRA, an advantage of CTA is the short examination time, which can be used for an emergency diagnosis of MMD in children. In addition, the sensitivity of CTA in describing the median sizes of arteries is better than that of MRA ${ }^{[55]}$. As with MRA, CTA is not only suitable for the diagnosis of MMD in children, it can also be used to evaluate the degree of angiogenesis after vascular bypass surgery [56].

\subsection{Cerebral perfusion imaging}

The cerebral blood flow volume of children with MMD is lower than that in healthy children of the same age. Additionally, the cerebral blood flow is mainly distributed in the posterior circulation, while cerebral blood flow is mainly distributed in the anterior circulation in healthy children. Therefore, the blood flow volume distribution plays an important role in the diagnosis of MMD in children [57]. Currently, common clinical examinations include single-photon emission computed tomography (SPECT), positron emission tomography (PET), computed tomography (CT) perfusion, and magnetic resonance (MR) perfusion, among others. Kim et al. used (68)Ga-Arg-Gly-Asp (RGD) PET to evaluate the degree of post surgical blood flow reconstruction in pediatric MMD patients [58]. Ancelet et al. determined the importance of CT perfusion and MR perfusion in evaluating disease severity and treatment efficacy [59]. 
Additionally, cerebral perfusion imaging has become increasingly important in the diagnosis and treatment of MMD in children. For example, important cerebral perfusion pressure parameters, such as the mean transit time, cerebral blood flow, and time to peak (TTP), can be used to compare the hemodynamic variations before and after vascular reconstruction [60]. For example, TJ Yun et al. found that in children with MMD, the total average TTP values were $3.35 \pm 2.53$ seconds and $1.73 \pm 1.53$ seconds before and after surgery, respectively. This result suggested a significant decrease in the TTP value and a remarkable improvement of cerebral blood flow after surgery, which reflects a positive outcome of the surgical treatment [61].

\subsection{EEG}

A hyperventilation-induced diffuse high voltage phase was observed in the EEG monitoring results for all children. This high voltage phase consisted of a single-phase slow wave characterized by a build up and termination at the end of hyperventilation. However, in children with MMD, $50 \%$ of patients demonstrated another high voltage phase after the end of hyperventilation. This high voltage phase was also a single-phase slow wave and was called the rebuild-up. This phenomenon might be caused by a decrease in the cerebral perfusion reserve, which can be considered a characteristic change in children with MMD [62,63]. However, the disadvantage of EEG is that it is unable to quantify detailed indices of the cerebral perfusion reserve.

\section{Clinical symptoms of MMD in chil- dren}

Maki et al. classified the clinical symptoms of MMD in children into four types: (1)bleeding type; (2)epileptic type; (3)infarction type; and (4)transient ischemic attack (TIA) type [64]. In contrast to adult MMD, ischemia is often observed at the onset of MMD in children. Therefore, the most common types of MMD in children are the TIA type and the infarction type, which account for $70-80 \%$ of MMD cases in children $[50,65]$.

The infarction type is more commonly observed than the TIA type in children with MMD. The main reason is that pediatric patients cannot accurately describe TIA symptoms; thus, the diagnosis is missed [62]. The ischemic damage can be classified as cortical, subcortical, and watershed type based on the location of the infarction. The watershed type is the most commonly observed type of ischemia in MMD in children, and the infarction is located in the deep watershed zone [66]. HJ Cho et al. found that cortical and the watershed types were the most common types of infarctions observed in children with MMD, while the honeycomb type was the most common type in adults with MMD [67].

MMD in children is also different from MMD in adults in terms of common symptoms such as headache, mental decline or degeneration, hypertension, and temporary or permanent blindness.

\section{Headache}

Headache is the most commonly observed symptom in children with MMD and is the most non-specific symptom. In addition, no effective clinical treatment has been discovered for this condition. A previous study revealed that possible reasons for headache occurrence might be cerebral hypoxia or an expanded pia mater in the collateral circulation, which could stimulate epidural pain receptors [13]. The treatment efficacy of this type of pain is often unsatisfactory. HJ Seol et al. evaluated 204 children with MMD and discovered that $25 \%$ of children with MMD presented headache symptoms. After indirect surgery, over $50 \%$ of patients still exhibited headache symptoms at least 12 months later [68].

\section{Mental decline or degeneration}

Mental decline is a specific symptom of MMD in children and is mainly due to the lack of cerebral perfusion over a long time period. Soriani et al. performed a 14-month follow-up study on MMD in children less than four years old and discovered that all of the children suffered from irreversible mental decline [69]. Kurokawa et al. found that four years after MMD onset, $92 \%$ of pediatric patients had normal intelligence levels. However, five to nine years later, only $40 \%$ of patients remained at normal intelligence levels, and after $10-15$ years, only $33 \%$ of pediatric MMD patients remained at normal levels [70]. It can be concluded that MMD has tremendous effects on the intelligence of children.

\section{Hypertension}

Hypertension is another common clinical symptom of children with MMD. A possible cause of this symptom could involve insufficient cerebral blood flow perfusion, resulting in a compensatory higher blood pressure in an effort to maintain a sufficient cerebral blood supply, which manifests as hypertension. Long-term hypertension can induce renal artery stenosis, which affects $10 \%$ of children with MMD [71].

\section{Temporary or permanent blindness}

Blindness in affected MMD patients is primarily a result of posterior circulation ischemia. Clinical reports that discuss this symptom are limited, but it is a prominent clinical manifestation of MMD in children. 
Specifically, the symptom appears as visual field hemianopia and a visual field defect. Miyamoto et al. evaluated 178 MMD patients and found that 48 patients had visual lesions; $79.1 \%$ of these patients were pediatric patients with MMD [72].

\section{Other symptoms}

In addition to common clinical symptoms, such as those discussed above, many non-specific clinical symptoms have also been observed, including hemiplegia, general paresis of the insane, loss of sensation, aphasia, and cognitive impairment, and the mechanisms might be related to ischemia in the frontal, parietal, and temporal lobes ${ }^{[64]}$. DS Kim et al. found that hemiplegia affected $86.4 \%$ of children with MMD [73]. $\mathrm{Hsu} \mathrm{YH}$ et al. discovered that cognitive impairment was related to temporal lobe lesions in children with MMD [74]. In addition, normal behaviors, including hyperventilation, crying, and coughing, are also suspected to be MMD-related symptoms in children [64].

\section{Treatment of MMD in children}

It is generally agreed that active surgical treatment should be provided for children with MMD. The purpose of surgical treatment is to establish external carotid artery collateral circulation by surgical means to prevent and treat ischemic damage in cerebral tissues [75]. Based on a previous report, the incidence of stroke in symptom-free MMD children is 3.2\% each year. If no active surgical treatment is applied, $37 \%$ of patients exhibit symptoms related to nervous system damage, and $3 \%$ of patients will die, which suggests the necessity of active surgical treatment in children with MMD [76]. Although conservative treatment is not the major treatment plan for MMD in children, it can be used as an auxiliary approach to surgical treatment. For example, the calcium channel blocker nicardipine exhibited a positive effect in improving the hemodynamics of children with MMD by optimizing the collateral circulation to prevent ischemia [77]. However, some medications, including corticosteroids, low-molecular-weight dextran, and antiplatelet drugs, have shown no significant effects on MMD in children based on clinical progression monitoring and imaging analysis ${ }^{[78]}$.

Surgical treatment is currently an effective method to treat MMD in children and includes direct and indirect surgeries.

\section{Direct surgery}

Direct surgery is primarily performed to directly anastomose the STA and MCA. To increase the success rate of this procedure, the cerebral blood flow volume is tested and is the primary factor when selecting the brain surface artery branches of the MCA
[79]. However, the current surgical success rate in children with MMD is not ideal for two major reasons: (1) the vessels on the cerebral surface are slender and fragile in children, and as a result, direct vascular reconstruction is much more difficult in children than in adults. (2) Due to the temporary blockage of the blood supply at the brain surface by the artery that is causing insufficient cerebral perfusion, cerebral tissue ischemia and infarction may occur and exacerbate the condition [11]. Various complications are often observed in many pediatric patients after surgery and result in poor prognoses. For example, Hayashi et al. found that some MMD pediatric patients showed various degrees of short-term deterioration of the nervous system after STA-MCA procedures [80]. Additionally, Robertson et al. observed blockage in vessels near the MCA after performing STA-MCA procedures in children with MMD [81]. These results might be due to the shift of the watershed zone after vascular reconstruction. Yoshida et al. completed a 78-month follow-up study on children with MMD after STA-MCA procedures and discovered that the STA was slender and thinner than before the surgery. Therefore, it can be inferred that, although direct surgery can achieve faster treatment efficacy than indirect surgery, these changes in the STA would affect the establishment of effective cerebral collateral circulation and could result in a reduced cerebral blood supply from the external carotid artery [82]. Ishii et al. found that only a limited area showed improvement in the cerebral blood flow after STA-MCA surgery. Although TIA symptoms could be controlled in some children with MMD after direct surgery, their mental disorders were exacerbated. This issue might be related to the fact that the frontal lobe circulation was not reconstructed after surgery [83]. Therefore, despite the significant effect of STA-MCA surgery in children, it is not able to completely alter the ischemic conditions in children with MMD and improve their prognosis.

\section{Indirect surgery}

The efficacy of indirect surgery in children with MMD is better than that in adults [84]. Takanashi et al. reported that, after indirect surgery, almost all children with MMD successfully established collateral circulation, while only $50 \%$ of adult MMD patients achieved this effect [50]. Compared to direct surgery, the advantages of indirect surgery include less trauma, easier technical requirements, and shorter surgical time, while a disadvantage is the longer period required for vascular reconstruction [85]. Therefore, indirect surgery is not recommended for emergencies that require immediate blood circulation improvement. For example, Miyamoto et al. suggested that 
indirect surgery is not recommended for treating MMD children with frequent TIA because it cannot rapidly accomplish vascular reconstructions [86]. Direct surgery can only improve the cerebral circulation in the area of the vascular reconstruction, while indirect surgery can improve the cerebral circulation on a relatively larger scale. Therefore, indirect surgery is extensively applied in children with MMD. Thines et al. showed that indirect surgery was favored in children with MMD due to its simplicity and good clinical results. In contrast, direct or preferentially combined surgery is more effective in adults with MMD for preventing the recurrence of ischemic or hemorrhagic stroke [85]. Currently, commonly used indirect surgical procedures in clinical practice include encephalomyosynangiosis (EMS), encephalo-duro-arteriosynangiosis (EDAS), encephalo-duro-myosynangiosis (EDMS), encephalo-duro-arterio-myosynangiosis (EDAMS), multiple burr hole surgery (MBHS), and transplantation of the greater omentum. However, there is no clinical evidence demonstrating which one of the above techniques is more effective in treating MMD in children [84,87].

\subsection{EMS, EDAS, EDMS and EDAMS}

EMS surgery involves attaching the temporal muscle directly onto the surface of the cerebral cortex to establish collateral circulation and is the first-line indirect surgical procedure ${ }^{[87]}$. EDAS involves freeing the superficial temporal artery, maintaining its complete patency, and then attaching it onto the brain surface. EDMS and EDAMS combine EMS and EDAS procedures to further improve these techniques. In theory, both are indirect vascular reconstruction procedures. In particular, EDAMS involves a variety of anatomical structures, such as the external dura mater, with its rich blood supply, the galea aponeurotica flap, and the temporal muscle and its deep temporal artery to promote postoperative coronary collateral circulation [88]. For EMS, EDAS, EDMS, and EDAMS, vascular reconstruction is restricted at the parietal center. The reason for this restriction is that the anterior branches of the STA are on the forehead and cannot participate in vascular reconstruction. Only the posterior branches of the STA are used in these procedures. Touho et al. suggested that the parietal branches of the STA have a limited blood supply area after surgery, which could not cover an extensive cortical functional area [89]. Due to the limitations discussed above, these surgical techniques are continuously being improved in clinical practice to increase the success rates of post-surgical vascular reconstruction procedures. Touho et al. discovered that the temporal muscle (especially calcified and thickened temporal muscle) oppressed the corresponding cere- bral tissues and induced brain ischemic symptoms during an EMS procedure. Therefore, the authors suggested prioritizing the scalp artery and the subcutaneous tissue of the corresponding collateral vessels to effectively treat mild hemiplegia and aphasia caused by ischemia in the MCA blood supply area. This procedure also improved the treatment of complications such as paraparesis and urine incontinence caused by ischemia in the ACA blood supply area [90]. Yoshida et al. suggested an incision in the arachnoid membrane during surgery. The reason for this step was that the incision could activate the establishment of collateral circulation, particularly by promoting anastomosis between the external carotid artery system and the brain's surface blood vessels [91]. Baaj et al. recommended overturning the dura mater near the middle meningeal artery because anastomosis was easily achieved between the exterior of the dura mater near the middle meningeal artery and the cerebral cortex [92].

\subsection{Other surgeries}

Pia mater anastomosis and MBHS. Pia mater anastomosis involves attaching the adventitia of the STA to the exposed pia mater after cutting open the dura mater and the arachnoid membrane [93]. The arachnoid membrane is incised during the surgery, and the dura mater is not blocked. However, the incidence of cerebrospinal leakage is extremely low, and the post-surgical effect is adequate [75]. For example, Robertson et al. performed the above surgery on 13 children with MMD, and 10 patients showed stabilized or improved conditions in the post-surgical follow-up [94]. MBHS involves drilling the skull, followed by an incision in the dura mater and opening of the arachnoid membrane and pia mater. The periosteum is then attached to the surface of the cerebral cortex. This surgery is an auxiliary procedure to STA-MCA, EMS, and EDAM procedures, among others. For example, Sainte-Rose et al. performed MBHS on 24 hemispheres of 14 children with MMD, resulting in no exacerbated ischemia outcomes and only one case with complications [95].

Transplantation of the greater omentum. This surgery involves transplanting the abdominal omentum to the surface of the cerebral cortex. The transplantation procedure directly anastomoses the gastroepiploic vessels in the omental free flap with the cerebral superficial temporal vessels. This procedure is rarely used in clinical practice and is suitable for MMD in children for whom direct and indirect surgeries are not possible [96]. The characteristics of this surgery include effectively treating ischemia in ACA and PCA blood supply area-induced MMD in children, while most procedures (e.g., STA-MCA, EMS, 
EDAS, EDMS, and others) can only treat ischemia in the MCA blood supply area-induced MMD in children[97]. In recent years, Navarro et al. have improved the success and post-surgical complication rates of this procedure using laparoscopy. They performed laparoscopic omental transplantation in three children with MMD, resulting in improved ischemic conditions within three months after the surgery. One year later, MRI examinations revealed significant enhancements in cerebral perfusion, and no new ischemic lesions were observed [96].

\section{Prognosis of MMD in children}

Evidence-based medicine has demonstrated that active surgical treatment for MMD in children can effectively increase the quality of life and reduce the risk of ischemic diseases. These improvements are primarily due to improved cerebral hemodynamics in patients. In contrast, conservative treatments resulted in poor prognoses in children with MMD [74]. For example, Imai $\mathrm{H}$ et al. examined 48 children with MMD who underwent surgical procedures and found that perioperative complications were observed in four of 48 patients. Moreover, except for one child with recurrent transient ischemic attacks, the patients showed excellent clinical outcomes ${ }^{[98]}$. Guzman et al. performed 168 surgeries on 96 children with MMD (76.2\% STA-MCA surgeries and $23.8 \%$ involving other indirect surgeries). Thirty days after surgery, only three patients had poor prognoses, whereas the other 93 patients had excellent prognoses [99]. Fujimura et al. performed STA-MCA surgery on 17 children with MMD; 14 patients had good prognoses (82.4\%), and none of the patients showed permanent functional nerve impairments [100]. Ross et al. performed EDAS on six children with MMD and reported the results from their 0.5- to 9-year follow-up periods. None of the patients exhibited evidence of deterioration due to the disease [64]. In conclusion, whether the surgical treatment is direct or indirect, the significance of active surgical treatment for children with MMD is affirmative. In most cases, surgical treatment results in satisfactory efficacy. Compared to adult patients, children with MMD can realize a good prognosis if early diagnosis and early active surgical treatment are achieved.

\section{Outlook of MMD in children}

For childhood-onset MMD, early diagnosis and treatment are crucial. However, the incapability of self-description in pediatric patients produces considerable difficulties for an early diagnosis. Therefore, based on the pathogenesis of MMD in children, the development of objective diagnostic approaches is extremely important. Considering the special ana- tomical and hemodynamic properties of children with MMD, the choice of a surgical plan and the judgment on prognosis are also important after surgery. Meanwhile, the application of precision instruments and monitoring hemodynamics during surgery can greatly assist in increasing the surgical success rate. In summary, increasing the cure rate of MMD in children requires long-term clinical studies and practice, which will, in turn, require the contributions and efforts of numerous excellent neurological surgeons.

\section{Competing Interests}

The authors have declared that no competing interest exists.

\section{References}

1. Takeuchi K, Shimizu K. Hypoplasia of the bilateral internal carotid arteries. Brain Nerve. 1957; 9:37-43.

2. Ganesan V. Moyamoya: to cut or not to cut is not the only question. A pediatric neurologist's perspective. Dev Med Child Neurol. 2010; 52:10-13.

3. Wakai K, Tamakoshi A, Ikezaki K, et al. Epidemiological features of moyamoya disease in Japan: findings from a nationwide survey. Clin Neurol Neurosurg. 1997; 99:S1-5.

4. Papavasiliou A, Bazigou-Fotopoulou H, Ikeda H. Familial Moyamoya disease in two European children. J Child Neurol. 2007; 22:1371-1376.

5. Uchino K, Johnston SC, Becker KJ, et al. Moyamoya disease in Washington State and California. Neurology. 2005; 65:956-958.

6. Baba T, Houkin K, Kuroda S. Novel epidemiological features of moyamoya disease. J Neurol Neurosurg Psychiatry. 2008; 79:900-904.

7. Kraemer M, Heienbrok W, Berlit P. Moyamoya disease in Europeans. Stroke. 2008; 39: 3193-3200.

8. Vimalesvaran S, Nachiappan N, Sithamparanathan Y. Moyamoya syndrome in a Malaysian child with Down syndrome. J Paediatr Child Health. 2013; 49:865-867.

9. Scott RM, Smith ER. Moyamoya disease and moyamoya syndrome. N Engl J Med. 2009;360:1226-1237.

10. Kikuchi M, Asato M, Sugahara S, et al. Evaluation of surgically formed collateral circulation in moyamoya disease with 3D-CT angiography: comparison with MR angiography and X-ray angiography. Neuropediatrics. 1996; 27:45-49.

11. Veeravagu A, Guzman R, Patil CG, et al. Moyamoya disease in pediatric patients: outcomes of neurosurgical interventions. Neurosurg Focus. 2008; 24:E16.

12. Baltsavias G, Khan N, Valavanis A. The collateral circulation in pediatric moyamoya disease. Childs Nerv Syst. 2015;31:389-398.

13. Kuroda S, Houkin K. Moyamoya disease: current concepts and future perspectives. Lancet Neurol. 2008; 7:1056-1066.

14. Yamashita M, Oka K, Tanaka K. Histopathology of the brain vascular network in moyamoya disease. Stroke. 1983;14:50-58.

15. Fukui M, Kono S, Sueishi K, et al. Moyamoya disease. Neuropathology. 2000;20:S61-64.

16. Ikeda H, Sasaki T, Yoshimoto T, et al. Mapping of a familial moyamoya disease gene to chromosome 3p24.2-p26. Am J Hum Genet. 1999;64:533-537.

17. Papavasiliou A, Bazigou-Fotopoulou H, Ikeda H. Familial Moyamoya disease in two European children. J Child Neurol. 2007;22:1371-1376.

18. Kang HS, Kim SK, Cho BK, et al. Single nucleotide polymorphisms of tissue inhibitor of metalloproteinase genes in familial moyamoya disease. Neurosurgery. 2006;58:1074-1080

19. Sakurai $\mathrm{K}$, Horiuchi $\mathrm{Y}$, Ikeda $\mathrm{H}$, et al. A novel susceptibility locus for moyamoya disease on chromosome 8q23. J Hum Genet. 2004;49:278-281.

20. Fujimura M, Sonobe S, Nishijima Y, et al. Genetics and Biomarkers of Moyamoya Disease: Significance of RNF213 as a Susceptibility Gene. J Stroke. 2014;16:65-72.

21. Hong SH, Wang KC, Kim SK, et al. Association of HLA-DR and -DQ Genes with Familial Moyamoya Disease in Koreans. J Korean Neurosurg Soc. 2009;46:558-563.

22. Park YS, Jeon YJ, Kim HS, et al. The role of VEGF and KDR polymorphisms in moyamoya disease and collateral revascularization. PLoS One. 2012;7:e47158.

23. Hitomi T, Habu T, Kobayashi H, et al. The moyamoya disease susceptibility variant RNF213 R4810K (rs112735431) induces genomic instability bymitotic abnormality. Biochem Biophys Res Commun. 2013;439:419-426.

24. Hitomi T, Habu T, Kobayashi H, et al. Down regulation of Securin by the variant RNF213 R4810K (rs112735431, G>A) reduces angiogenic activity ofinduced pluripotent stem cell-derived vascular endothelial cells from moyamoya patients. Biochem Biophys Res Commun. 2013;438:13-19. 
25. Hyakuna N, Muramatsu $\mathrm{H}$, Higa T, et al Germline mutation of CBL is associated with moyamoya disease in a child with juvenile myelomonocytic leukemiaand Noonan syndrome-like disorder. Pediatr Blood Cancer. 2015;62:542-544.

26. Darrigo Júnior LG, Valera ET, Machado Ade A, et al. Moyamoya syndrome associated with neurofibromatosis type I in a pediatric patient. Sao Paulo Med J. 2011;129:110-112.

27. Park YS, Min KT, Kim TG, et al. Age-specific eNOS polymorphisms in moyamoya disease. Childs Nerv Syst. 2011;27:1919-1926.

28. Sakamoto S, Kiura Y, Yamasaki F, et al. Expression of vascular endothelial growth factor in dura mater of patients with moyamoya disease. Neurosurg Rev. 2008;31:77-81.

29. Yoshimoto T, Houkin K, Takahashi A, et al. Angiogenic factors in moyamoya disease. Stroke. 1996;27:2160-2165.

30. Hojo M, Hoshimaru M, Miyamoto S, et al. Role of transforming growth factor-beta1 in the pathogenesis of moyamoya disease. J Neurosurg. 1998;89:623-629.

31. Greco F, Castellano Chiodo D, Sorge A, et al. Multiple arterial ischemic strokes in a child with moyamoya disease and Mycoplasma pneumoniae infection. Minerva Pediatr. 2006;58:63-68

32. Yamada H, Deguchi K, Tanigawara T, et al. The relationship between moyamoya disease and bacterial infection. Clin Neurol Neurosurg. 1997;99:S221-224.

33. Sharfstein SR, Ahmed S, Islam MQ et al. Case of moyamoya disease in a patient with advanced acquired immunodeficiency syndrome. J Stroke Cerebrovasc Dis. 2007;16:268-272.

34. Hsiung GY, Sotero de Menezes M. Moyamoya syndrome in a patient with congenital human immunodeficiency virus infection. I Child Neurol. 1999;14:268-270

35. Kim TK, Eun BL, Cha SH, et al. Moyamoya disease in a child with previous acute necrotizing encephalopathy. Pediatr Radiol. 2003;33:644-647.

36. $\mathrm{Li} \mathrm{H}$, Zhang ZS, Dong ZN, et al. Increased thyroid function and elevated thyroid autoantibodies in pediatric patients with moyamoya disease: acase-control study. Stroke. 2011;42:1138-1139.

37. Kansal R, Mahore A, Nadkarni T, et al. Fetal radiation exposure and moyamoya disease. J Clin Neurosci. 2010;17:406-407.

38. Kikuchi A, Maeda M, Hanada R, et al; Tokyo Children's Cancer Study Group (TCCSG). Moyamoya syndrome following childhood acute lymphoblastic leukemia. Pediatr Blood Cancer. 2007;48:268-272.

39. Ullrich NJ, Robertson R, Kinnamon DD, et al. Moyamoya following cranial irradiation for primary brain tumors in children. Neurology. 2007:68:932-938.

40. Kestle JR, Hoffman HJ, Mock AR. Moyamoya phenomenon after radiation for optic glioma. J Neurosurg. 1993;79:32-35.

41. Ni J, Zhou LX, Wei YP, et al. Moyamoya syndrome associated with Graves' disease: a case series study. Ann Transl Med. 2014·2·77.

42. Delavari N, Strahle J, Maher CO. Moyamoya syndrome associated with hemoglobin Southampton (Casper). Pediatr Neurosurg. 2013;49:307-310.

43. Han C, Yang WZ, Zhang HT,et al. Clinical characteristics and long-term outcomes of moyamoya syndrome associated with neurofibromatosis type 1 . J Clin Neurosci. 2015;22:286-290

44. Buchbinder D, Steinberg G, Linetsky M, et al. Moyamoya in a child treated with interferon for recurrent osteosarcoma. J Pediatr Hematol Oncol. 2010:32:476-478.

45. Meena SS, Ramkumar TV, Sharma S, et al. Moyamoya syndrome associated with severe iron deficiency anemia in a young child. Pediatr Hematol Oncol. 2012:29:368-371.

46. Vo Van P, Sabouraud P, Mac G, et al. Moyamoya disease associated with hereditary spherocytosis. Pediatr Neurol. 2011;44:69-71.

47. Ramesh K, Sharma S, Raju V, et al. Renal agenesis and external iliac artery stenosis in an infant with moyamoya disease. Brain Dev. 2011;33:612-615.

48. Fukui M. Guidelines for the diagnosis and treatment of spontaneous occlusion of the circle of Willis ('moyamoya' disease). Research Committee on Spontaneous Occlusion of the Circle of Willis (Moyamoya Disease) of the Ministry of Health and Welfare, Japan. Clin Neurol Neurosurg. 1997;99:S238-240.

49. Kim JE, Jeon JS. An update on the diagnosis and treatment of adult Moyamoya disease taking into consideration controversial issues. Neurol Res. 2014;36:407-416

50. Takanashi J. Moyamoya disease in children. Brain Dev. 2011;33:229-234.

51. Matsushima T, Inoue T, Suzuki SO, et al. Surgical treatment of moyamoya disease in pediatric patients--comparison between the results of indirect and direct revascularization procedures. Neurosurgery. 1992;31:401-405.

52. Kikuchi M, Asato M, Sugahara S, et al. Evaluation of surgically formed collateral circulation in moyamoya disease with 3D-CT angiography: comparison with MR angiography and X-ray angiography. Neuropediatrics. 1996;27:45-49.

53. Yamada I, Suzuki S, Matsushima Y. Moyamoya disease: comparison of assessment with MR angiography and MR imaging versus conventional angiography. Radiology. 1995;196:211-218.

54. Houkin K, Nakayama N, Kuroda S, et al. How does angiogenesis develop in pediatric moyamoya disease after surgery? A prospective study with MR angiography. Childs Nerv Syst. 2004; 20: 734-741.

55. Currie S, Raghavan A, Batty R, et al. Childhood moyamoya disease and moyamoya syndrome: a pictorial review. Pediatr Neurol. 2011:44:401-413.

56. Sugino T, Mikami T, Ohtaki S, et al. Assessment of moyamoya disease using multidetector row computed tomography. J Stroke Cerebrovasc Dis. 2013;22:644-649.
57. Song YS, Oh SW, Kim YK, et al. Hemodynamic improvement of anterior cerebral artery territory perfusion induced by bifrontal encephalo(periosteal) synangiosis in pediatric patients with moyamoya disease: a study with brain perfusion SPECT. Ann Nucl Med. 2012;26:47-57.

58. Kim YI, Phi JH, Paeng JC, et al. In vivo evaluation of angiogenic activity and its correlation with efficacy of indirect revascularization surgery inpediatric moyamoya disease. J Nucl Med. 2014;55:1467-1472.

59. Ancelet C, Boulouis G, Blauwblomme T, et al. Imaging Moya-Moya disease. Rev Neurol (Paris). 2014;30. pii: S0035-3787(14)01057-1.

60. Togao O, Mihara F, Yoshiura T, et al. Cerebral hemodynamics in Moyamoya disease: correlation between perfusion-weighted MR imaging and cerebral angiography. AJNR Am J Neuroradiol. 2006;27:391-397.

61. Yun TJ, Cheon JE, Na DG, et al. Childhood moyamoya disease: quantitative evaluation of perfusion MR imaging--correlation with clinical outcome after revascularization surgery. Radiology. 2009;251:216-223.

62. Ibrahimi DM, Tamargo RJ, Ahn ES. Moyamoya disease in children. Childs Nerv Syst. 2010;26:1297-1308.

63. Cho A, Chae JH, Kim HM, et al. Electroencephalography in pediatric moyamoya disease: reappraisal of clinical value. Childs Nerv Syst. 2014;30:449-459.

64. Maki Y, Enomoto T. Moyamoya disease. Childs Nerv Syst. 1988:4:204-212.

65. Yonekawa Y. Operative neurosurgery: personal view and historical backgrounds (9) Moyamoya angiopathy (MMA): past history and status presens. No Shinkei Geka. 2012;40:67-87.

66. Rafay MF, Armstrong D, Dirks P, et al. Patterns of cerebral ischemia in children with moyamoya. Pediatr Neurol. 2015;52:65-72.

67. Cho HJ, Jung YH, Kim YD, et al. The different infarct patterns between adulthood-onset and childhood-onset moyamoya disease. J Neurol Neurosurg Psychiatry. 2011;82:38-40.

68. Seol HJ, Wang KC, Kim SK, et al. Headache in pediatric moyamoya disease: review of 204 consecutive cases. J Neurosurg. 2005;103: 439-442.

69. Soriano SG, Cowan DB, Proctor MR, et al. Levels of soluble adhesion molecules are elevated in the cerebrospinal fluid of children with moyamoya syndrome. Neurosurgery. 2002;50:544-549.

70. Kurokawa T, Tomita S, Ueda K, et al. Prognosis of occlusive disease of the circle of Willis (moyamoya disease) in children. Pediatr Neurol. 1985;1:274-277.

71. Yamada I, Himeno $Y$, Matsushima $Y$, et al. Renal artery lesions in patients with moyamoya disease: angiographic findings. Stroke. 2000;31:733-737.

72. Miyamoto S, Kikuchi H, Karasawa J, et al. Study of the posterior circulation in moyamoya disease. Part 2: Visual disturbances and surgical treatment. J Neurosurg. 1986;65:454-460.

73. Kim DS, Ko TS, Ra YS, et al. Postoperative electroencephalogram for follow up of pediatric Moyamoya disease. J Korean Med Sci. 2006;21:495-499.

74. Hsu YH, Kuo MF, Hua MS, et al. Selective neuropsychological impairments and related clinical factors in children with moyamoya disease of the transient ischemic attack type. Childs Nerv Syst. 2014;30:441-447.

75. Scott RM, Smith JL, Robertson RL, et al. Long-term outcome in children with moyamoya syndrome after cranial revascularization by pial synangiosis. J Neurosurg. 2004;100:142-149.

76. Ng J, Thompson D, Lumley JP, et al. Surgical revascularisation for childhood moyamoya. Childs Nerv Syst. 2012;28:1041-1048.

77. Hosain SA, Hughes JT, Forem SL, et al. Use of a calcium channel blocker (nicardipine $\mathrm{HCl}$ ) in the treatment of childhood moyamoya disease. J Child Neurol. 1994:9:378-380.

78. Scott RM. Surgical treatment of moyamoya syndrome in children. Pediatr Neurosurg 1995;22:39-48

79. Takahashi A, Kamiyama H, Houkin K, et al. Surgical treatment of childhood moyamoya disease--comparison of reconstructive surgery centered on the frontal region and the parietal region. Neurol Med Chir (Tokyo). 1995;35:231-237.

80. Hayashi T, Shirane R, Fujimura M, et al. Postoperative neurological deterioration in pediatric moyamoya disease: watershed shift and hyperperfusion. J Neurosurg Pediatr. 2010;6:73-81.

81. Robertson RL, Burrows PE, Barnes PD, et al. Angiographic changes after pial synangiosis in childhood moyamoya disease. AJNR Am J Neuroradiol. 1997:18:837-845

82. Yoshida YK, Shirane R, Yoshimoto T. Non-anastomotic bypass surgery for childhood moyamoya disease using dural pedicle insertion over the brain surface combined with encephalogaleomyosynangiosis. Surg Neurol. 1999;51:404-411

83. Ishii $\mathrm{R}$, Takeuchi $\mathrm{S}$, Ibayashi $\mathrm{K}$, et al. Intelligence in children with moyamoya disease: evaluation after surgical treatments with special reference tochanges in cerebral blood flow. Stroke. 1984;15:873-877.

84. Abla AA, Gandhoke G, Clark JC, et al. Surgical outcomes for moyamoya angiopathy at barrow neurological institute with comparison of adult indirect encephaloduroarteriosynangiosis bypass, adult direct superficial temporal artery-to-middle cerebral artery bypass, and pediatric bypass: 154 revascularization surgeries in 140 affected hemispheres. Neurosurgery. 2013:73:430-439.

85. Thines L, Petyt G, Aguettaz P, et al. Surgical management of Moyamoya disease and syndrome: Current concepts and personal experience. Rev Neurol (Paris). 2015;171:31-44.

86. Miyamoto S, Kikuchi H, Karasawa J, et al. Pitfalls in the surgical treatment of moyamoya disease. Operative techniques for refractory cases. J Neurosurg. $1988 ; 68: 537-543$ 
87. Wang KC, Phi JH, Lee JY, et al. Indirect revascularization surgery for moyamoya disease in children and its special considerations. Korean J Pediatr. 2012;55:408-413.

88. Kim DS, Kang SG, Yoo DS, et al. Surgical results in pediatric moyamoya disease: angiographic revascularization and the clinical results. Clin Neurol Neurosurg. 2007;109:125-131.

89. Touho H, Karasawa J, Ohnishi $\mathrm{H}$, et al. Surgical reconstruction of failed indirect anastomosis in childhood Moyamoya disease. Neurosurgery. 1993;32:935-940.

90. Touho H. Subcutaneous tissue graft including a scalp artery and a relevant vein for the treatment of cerebral ischemia in childhood moyamoya disease. Surg Neurol. 2007;68:639-645.

91. Yoshida YK, Shirane R, Yoshimoto T. Non-anastomotic bypass surgery for childhood moyamoya disease using dural pedicle insertion over the brain surface combined with encephalogaleomyosynangiosis. Surg Neurol. 1999;51:404-411.

92. Baaj AA, Agazzi S, Sayed ZA, et al. Surgical management of moyamoya disease: a review. Neurosurg Focus. 2009;26:E7.

93. Adelson PD, Scott RM. Pial synangiosis for moyamoya syndrome in children. Pediatr Neurosurg.1995;23:26-33.

94. Robertson RL, Burrows PE, Barnes PD, et al. Angiographic changes after pial synangiosis in childhood moyamoya disease. AJNR Am J Neuroradiol. 1997;18:837-845.

95. Sainte-Rose C, Oliveira R, Puget $S$, et al. Multiple bur hole surgery for the treatment of moyamoya disease in children. J Neurosurg. 2006;105:437-443.

96. Navarro R, Chao K, Gooderham PA, et al. Less invasive pedicled omental-cranial transposition in pediatric patients with moyamoya disease and failed prior revascularization. Neurosurgery. 2014;10:1-14.

97. Ohtaki M, Uede $\mathrm{T}$, Morimoto $\mathrm{S}$, et al. Intellectual functions and regional cerebral haemodynamics after extensive omental transplantation spread over both frontal lobes in childhood moyamoya disease. Acta Neurochir (Wien). 1998;140:1043-1053.

98. Imai $\mathrm{H}$, Miyawaki $\mathrm{S}$, Ono $\mathrm{H}$, et al. The importance of encephalo-myo-synangiosis in surgical revascularization strategies for Moyamoya Disease in children and adults. World Neurosurg. 2015.

99. Guzman R, Lee M, Achrol A, et al. Clinical outcome after 450 revascularization procedures for moyamoya disease. Clinical article. J Neurosurg. 2009;111:927-935.

100. Fujimura M, Kaneta T, Tominaga T. Efficacy of superficial temporal artery-middle cerebral artery anastomosis with routine postoperative cerebral blood flow measurement during the acute stage in childhood moyamoya disease. Childs Nerv Syst. 2008;24:827-832. 